\title{
Effects of Heart Rate on the Pump Function and Electrophysiological Characteristics of the Heart in the Frog Rana temporaria
}

\author{
Natalya A. Kibler, $\mathrm{PhD}^{1 *}$; Vladimir P. Nuzhny, $\mathrm{PhD}, \mathrm{ScD}^{1}$; Svetlana V. Achmetzhynova ${ }^{2}$; \\ Dmitry N. Shmakov ${ }^{1}$ \\ ${ }^{1}$ Institute of Physiology, Komi Science Center, Urals Branch of the RAS \\ ${ }^{2}$ Syktyvkar State University named after Pitirim Sorokin \\ Syktyvkar, Komi Republic, the Russian Federation
}

\begin{abstract}
The aim of the study was to investigate the electrical activity and contractility of the heart ventricle in frogs Rana temporaria $(\mathrm{n}=14)$ under different heart rates. The activation time (AT, as $\mathrm{dV} / \mathrm{dt}_{\min }$ during QRS complex), the repolarization time (RT, as $\mathrm{dV} / \mathrm{dt}_{\max }$ during ST-T wave), and the activation-recovery intervals (ARIs, as difference between RT and AT) were measured. The hemodynamic variables were determined with the Prucka MacLab 2000 system. Heart rate (HR) was changed by the use of right atrium pacing from $0.6 \mathrm{~Hz}$ to $1.1 \mathrm{~Hz}$ with step $0.1 \mathrm{~Hz}$. The increasing $\mathrm{HR}$ from $0.6 \mathrm{~Hz}$ to $1.1 \mathrm{~Hz}$ led to the increased duration of ARIs on the ventral and dorsal fragments of ventricular epicardium as compared with initial sinus rhythm. During the high HR, more prolonged ARIs were observed on the ventral side of the epicardium than on the dorsal surface (exclusion is supraventricular rhythm with rate of $1.1 \mathrm{~Hz}$ ). The repolarization dispersion of epicardium on the whole, as well as repolarization of both epicardial sides separately, decreased under the higher rate. Repolarization sequence depended on the activation sequence and the distribution of local repolarization durations only at supraventricular rhythm with a frequency of $1.1 \mathrm{~Hz}$. The indexes of pump function decreased under high HR. Thus, the increased HR resulted in a decrease in the dispersion of repolarization and ARIs; the repolarization duration of ventricular epicardium at supraventricular rhythms was shortened as compared with sinus rhythm. During an increase in HR, repolarization sequence is formed in association with the level of ARI dispersion and changes of the repolarization duration. (Int J Biomed. 2017; 7(1):46-50.)
\end{abstract}

Key words: ventricular pump function $\bullet$ heart rate $\bullet$ repolarization $\bullet$ activation-recovery intervals $\bullet$ frog Rana temporaria.

\section{Abbreviations}

CO, cardiac output; EDP, end-diastolic pressure; HR, heart rate; $\mathbf{L V}$, left ventricle; $\mathbf{L A}$, left atrium; RA, right atrium; $\mathbf{S V}$, stroke volume; VE, ventricular epicardium; VEDV, ventricular end-diastolic volume.

\section{Introduction}

The structural and hemodynamic changes during the evolution of the vertebrate heart are not reflected in obvious changes in the overall electrical patterning of the heart. ${ }^{[1]}$ The total duration of the electrical events of a given heart beat is shorter in endothermic birds and mammals with a high

*Corresponding author: Natalya A. Kibler., PhD; Laboratory of Cardiac Physiology, Institute of Physiology, Komi Science Center, Ural Branch of the RAS, Syktyvkar, Komi Republic, Russia. E-mail: natanadya@mail.ru
HR ${ }^{[1,2]}$ than in ectothermic vertebrates. ${ }^{[3,4]}$ This difference in $\mathrm{HR}$ is connected with a low degree evolutionary development of cardiac conduction system (CCS) among groups of poikilotherm vertebrates, ${ }^{[5,1]}$ as a result, ectothermic animals are unable to develop a high HR in nature. ${ }^{[1]}$

High HR leads to the mechanical changes of the indexes of cardiac pump function in endothermic animals. Early investigations showed that rising HR resulted in the elevation of LV preload and pressure in LA and a decrease in systolic LV pressure and isovolumic indexes $\left(\mathrm{dP} / \mathrm{dt}_{\max }, \mathrm{dP} / \mathrm{dt}_{\min }\right), \mathrm{LVEDV}$ and $\mathrm{CO}$ in dogs. ${ }^{[6,7]}$ However, an increased HR resulted in increasing the isovolumic indexes ${ }^{[8]}$ and decreasing $\mathrm{SV}$, 
LVEDV, and LV preload in pigs. ${ }^{[9]}$ In the lowest vertebrates, a functional organization of the ventricular myocardium under electrical stimulation at different frequencies has not been sufficiently studied. It should be noted that early data about pump function and electrical properties were based on specific anatomo-physiological cardiac characteristics in amphibians (presence of the single ventricle, poor development of CCS, sequential type of the activation of ventricular myocardium, low HR and others) in conditions of natural habitat.

The dependence of heart contractility on $\mathrm{HR}^{[10]}$ is a very important cardiac inotropic mechanism of the majority of species of animals; therefore, amphibians are a convenient model for research of the features of changes in the electrical properties and cardiac contractile function. The study of the electrophysiological and contractile properties of the ventricle in ectothermic vertebrates (amphibians) with specific structural cardiac organization is necessary for understanding the patterns of cardiac evolution and adaptation of the heart to different habitats of these animals. The analysis of the electrophysiological and hemodynamic cardiac properties under different HRs will promote the further clarification of the adaptation mechanisms of the lowest ectothermic vertebrates to changing environmental conditions.

The aim of the study was to investigate the electrical activity and contractility of the heart ventricle in frogs Rana temporaria under different HRs.

\section{Material and methods}

\subsection{Animals and surgical procedure}

The investigation conforms with the Guide for the Care and Use of Laboratory Animals published by the US National Institutes of Health (NIH Publication No. 85-23, revised 1996). Experiments were performed on 14 adult (2-3 years old) frogs (Rana temporaria) of both sexes weighing from $34 \mathrm{~g}$ to $47 \mathrm{~g}$. All animals demonstrated normal sinus rhythm on an ECG. The frogs were anaesthetized by placing them for 3 minutes in a jar containing $40 \%$ ethanol. After that the ventral thoracic wall was removed and the pericardium was cut open.

During the experiment, the body temperature of each animal was in the range of 18 to $20^{\circ} \mathrm{C}$ and the heart was flushed with warm Ringer's solution $\left(18-20^{\circ} \mathrm{C}\right)$. In the laboratory, their body temperature equilibrated rapidly with the ambient temperature that corresponds to the data of other researchers. At the end of the experiment, the animals were euthanized by the intravenous injection of an overdose of alcoholic solution.

\subsection{Hemodynamic recording}

The hemodynamic variables were determined with the Prucka MacLab 2000 system (GE Medical System, GmbH). The pressure in the ventricle was measured with a catheter (internal diameter, $1 \mathrm{~mm}$ ) filled with the heparinized $0.9 \%$ saline inserted via the free wall into the ventricle cavity. Invasive monitoring of the pressure was carried out using transducers, transforming blood pressure inside of the vessels as the transducer registered mechanical changes.

Heart performance was evaluated as follows: inotropism was evaluated in terms of maximal systolic ventricular pressure (MSVP in $\mathrm{mmHg}$, index of contractile activity) and maximal value of the MSVP derivative $\left[+(\mathrm{dP} / \mathrm{dt})_{\max }, \mathrm{mmHg} / \mathrm{s}\right.$; index of maximal ventricle contraction rate]]. Lusitropism was assessed on the basis of the maximal rate of MSVP decline [$\left.\left.(\mathrm{dP} / \mathrm{dt})_{\min }, \mathrm{mmHg} / \mathrm{s}\right]\right]$ and $\mathrm{EDP}(\mathrm{mmHg})$.

\subsection{Electrocardiographic recording and analysis}

Standard bipolar limb lead electrocardiograms were recorded with an application of subcutaneous steel needle electrodes. Registration of an epicardial electrogram was performed by using a matrix $(5 \mathrm{~mm} \times 5 \mathrm{~mm})$ containing 64 electrodes at the sinoatrial and supraventricular rhythms. The matrix was alternately superimposed on the central portion of the ventral and dorsal sides of the epicardium, in such a way that the cephalic border of the matrix grasped the basal part and the inferior border - part of the ventricular apex.

In every epicardial lead, the activation time (AT), the repolarization time (RT), and the activation-recovery intervals (ARIs) were obtained. The latter was used to assess local repolarization durations. AT, RT and ARIs were determined as $\mathrm{dV} / \mathrm{dt}_{\text {min }}$ during QRS complex, $\mathrm{dV} / \mathrm{dt}_{\text {max }}$ during ST-T wave, and the difference between RT and AT, respectively. ${ }^{[15]}$ The values were determined automatically, inspected by the observer and corrected manually if necessary. In each set of simultaneously recorded electrograms, the beginning of the QRS complex in the II limb lead was chosen as a reference time point with respect to which ATs and RTs were measured in a given set of electrograms.

The dispersion of ATs, ARIs, and RTs of the ventricle were taken as the difference between the maximal and minimal AT, RT and ARI values in a set of recorded electrograms, respectively.

In order to construct isochronal activation maps, the zero point was assigned to the timing of the epicardial activation breakthrough. Similarly, the zero points in the repolarization maps identify for the earliest repolarization on the epicardium. At the end of the experiment, the heart was removed for weight measurements.

\subsection{Stimulation}

HR was changed by the use of right atrium pacing from 0.6 to $1.1 \mathrm{~Hz}$ with step $0.1 \mathrm{~Hz}$. RA was stimulated (supraventricular rhythm) with cathodic impulses (duration of $1 \mathrm{~ms}$; twice diastolic threshold) at a cycle length $1200 \mathrm{~ms}$. Amplitude of impulses was 4-8 V. The duration of the pacing period was $1 \mathrm{~min}$.

2.5. Statistical examination was done using statistical package Primer of Biostatics 4.03 and SPSS 11.5 using Wilcoxon test for paired comparisons and Fridman test followed by the Wilcoxon test with Bonferroni correction for multiple comparisons. Values are given as $\mathrm{M} \pm \mathrm{SD}$. A probability value of $P<0.05$ was considered statistically significant.

\section{Results}

\section{Ventricular epicardial activation, repolarization and ARIS} under the spontaneous sinus rhythm

At the sinus rhythm, HR was $38 \pm 7 \mathrm{bpm}$ and duration of QRS complex - 78.2 $\pm 7.4 \mathrm{~ms}$. The depolarization wave of VE on the ventral and dorsal parts spread from the base (20.4 \pm 3.7 
$\mathrm{ms}$ and $27.4 \pm 2.1 \mathrm{~ms}$, respectively) to apex $(36.6 \pm 6.0 \mathrm{~ms}$ and $42.2 \pm 7.3 \mathrm{~ms} ; P<0.05)$, and from the right $(24.5 \pm 4.2 \mathrm{~ms})$ to the left $(38.6 \pm 5.3 \mathrm{~ms} ; P<0.05)$ on the ventral side.

Repolarization sequence on the ventricular epicardial surface of both sides was similar to the activation sequence; however, the apicobasal gradient was not statistically significant. Under sinus rhythm, dispersions of activation and repolarization increased in the dorsal-to-ventral direction $(P<0.05)$ (Table 1). With that, ARIs were increased on the ventral side of VE as compared with dorsal side $(P<0.05)$. On the ventral side, ARIs were significantly longer on the left part of VE than on its right part (894.8 $\pm 204.2 \mathrm{~ms}$ vs. $813.2 \pm 193.0$ $\mathrm{ms} ; P<0.05)$. Dispersion of ARIs was increased in the dorsalto-ventral direction.
II. Hemodynamics, ventricular epicardial activation, repolarization and ARIs under the electrical stimulation of $R A$

Duration of the QRS complex did not significantly change under the increase in HR from $0.6 \mathrm{~Hz}$ to $1.1 \mathrm{~Hz}$, but the duration of the QT interval was decreased as compared with initial sinus rhythm. The indexes of the pump function ( $\mathrm{SPV}, \mathrm{dP} / \mathrm{dt}_{\max }$, and $\mathrm{dP} / \mathrm{dt}_{\min }$ ) are presented in Table 2. During RA pacing $(1.1 \mathrm{~Hz})$, the activation dispersion (duration of activation) was increased $(P<0.05)$ on the dorsal side of $\mathrm{VE}$ (Table 1). Under the increase in HR from $0.6 \mathrm{~Hz}$ to $0.9 \mathrm{~Hz}$, the difference in time depolarization was insignificant between the ventral and dorsal sides of VE. However, during the next increase in HR up to $1.1 \mathrm{~Hz}$, the ventral side was depolarizated earlier as compared with dorsal side $(P<0.05)$

Table 1.

Effect of HR on the dispersion of AT, ARI, and RT ( $n=14)$

\begin{tabular}{|c|c|c|c|c|c|c|c|c|}
\hline \multirow{2}{*}{\multicolumn{2}{|c|}{ Parameters }} & \multirow{2}{*}{$\begin{array}{l}\text { Sinus } \\
\text { rhythm }\end{array}$} & \multicolumn{6}{|c|}{ HR (Hz) / Duration of cardiac cycle (ms) } \\
\hline & & & $0.6 / 1570$ & $0.7 / 1360$ & $0.8 / 1250$ & $0.9 / 1140$ & $1.0 / 1000$ & $1.1 / 937$ \\
\hline \multicolumn{2}{|r|}{$\mathrm{HR}, \mathrm{bpm}$} & $38 \pm 7$ & 38 & 44 & 48 & 55 & 60 & 64 \\
\hline 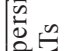 & Ventral side & $97.4 \pm 18.7^{\wedge}$ & $80.6 \pm 23.6$ & $105.4 \pm 32.2$ & $63.4 \pm 21.4$ & $94.9 \pm 25.3$ & $74.2 \pm 11.0$ & $70.7 \pm 20.3^{\wedge}$ \\
\hline 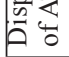 & Dorsal side & $73.3 \pm 25.3$ & $70.3 \pm 20.3$ & $87.1 \pm 16.9$ & $95.6 \pm 22.4$ & $67.3 \pm 18.4$ & $55.4 \pm 11.3$ & $106.8 \pm 29.2 *$ \\
\hline 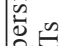 & Ventral side & $600.2 \pm 113.7^{\wedge}$ & $627.7 \pm 153.6$ & $501.1 \pm 107.4$ & $215.2 \pm 53.4 *$ & $293.5 \pm 72.2 *$ & $194.7 \pm 85.8^{*}$ & $204.6 \pm 103.2 *$ \\
\hline $\begin{array}{ll}5 \\
0\end{array}$ & Dorsal side & $569.6 \pm 94.4$ & $576.4 \pm 105.9$ & $429.5 \pm 102.6$ & $215.6 \pm 89.0^{*}$ & $285.7 \pm 96.8^{*}$ & $211.0 \pm 81.7^{*}$ & $277.6 \pm 88.3 *$ \\
\hline \multirow{3}{*}{ 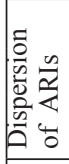 } & GVD & $599.2 \pm 133.7$ & $451.6 \pm 106.4$ & $452.4 \pm 153.6$ & $326.2 \pm 111.7$ & $295.2 \pm 82.0 *$ & $217.3 \pm 73.4^{*}$ & $259.4 \pm 109.6^{*}$ \\
\hline & \begin{tabular}{|l} 
Ventral side \\
\end{tabular} & $611.1 \pm 109.6^{\wedge}$ & $408.3 \pm 102.2$ & $322.1 \pm 92.0$ & $355.4 \pm 104.5$ & $293.5 \pm 84.9^{*}$ & $184.7 \pm 44.9^{*}$ & $203.0 \pm 51.1^{*}$ \\
\hline & Dorsal side & $585.0 \pm 106.7$ & $484.3 \pm 117.1$ & $354.0 \pm 107.2$ & $322.1 \pm 110.9$ & $299.4 \pm 74.5^{*}$ & $242.6 \pm 73.7^{*}$ & $315.7 \pm 72.9 *$ \\
\hline$\vec{\theta}<$ & Dorsal side & $772.2 \pm 101.1$ & $593.4 \pm 107.1^{*}$ & $492.1 \pm 94.0 *$ & $463.0 \pm 112.4 *$ & $291.4 \pm 100.9 *$ & $269.3 \pm 104.0 *$ & $288.7 \pm 95.0 *$ \\
\hline
\end{tabular}

GVD - General ventricular dispersion; GDV ARI - General duration ventricular ARI; ^ $P<0.05$ values vs dorsal side; $* P<0.05$ values vs sinus rhythm.

Table 2.

Haemodynamic indexes, electric parameters and cardiac mass under the increase in HR

\begin{tabular}{|l|c|c|c|c|c|c|c|}
\hline \multirow{2}{*}{ Parameters } & \multirow{2}{*}{$\begin{array}{c}\text { Sinus } \\
\text { rhythm }\end{array}$} & \multicolumn{6}{|c|}{ HR (Hz) / Duration of cardiac cycle (ms) } \\
\cline { 3 - 8 } & $0.6 / 1570$ & $0.7 / 1360$ & $0.8 / 1250$ & $0.9 / 1140$ & $1.0 / 1000$ & $1.1 / 937$ \\
\hline $\mathrm{HR}, \mathrm{bpm}$ & $38 \pm 7$ & 38 & 44 & 48 & 55 & 60 & 64 \\
\hline $\mathrm{MSVP}, \mathrm{mmHg}$ & $24.3 \pm 3.4$ & $18.5 \pm 3.4^{*}$ & $16.2 \pm 5.0^{*}$ & $15.4 \pm 5.3^{*}$ & $14.6 \pm 4.8^{*}$ & $14.7 \pm 5.9^{*}$ & $14.4 \pm 5.3^{*}$ \\
\hline $\mathrm{EDP}, \mathrm{mmHg}$ & $1.7 \pm 0.5$ & $1.0 \pm 0.2$ & $1.1 \pm 0.5$ & $1.1 \pm 0.6$ & $1.3 \pm 0.4$ & $1.4 \pm 0.2$ & $1.4 \pm 0.3$ \\
\hline $\begin{array}{l}+\mathrm{dP} / \mathrm{dt}_{\text {max }} \\
\mathrm{mmH} / \mathrm{s}\end{array}$ & $126.5 \pm 36.7$ & $83.2 \pm 16.7^{*}$ & $83.4 \pm 20.3^{*}$ & $83.2 \pm 21.9^{*}$ & $82.2 \pm 19.8^{*}$ & $81.1 \pm 16.6^{*}$ & $73.7 \pm 17.9^{*}$ \\
\hline $\begin{array}{l}-\mathrm{dP} / \mathrm{dt}_{\text {min }} \\
\mathrm{mmHg} / \mathrm{s}\end{array}$ & $110.2 \pm 38.7$ & $86.2 \pm 21.7^{*}$ & $83.4 \pm 18.5^{*}$ & $68.2 \pm 13.5^{*}$ & $67.2 \pm 15.4^{*}$ & $61.2 \pm 17.6^{*}$ & $61.7 \pm 17.8^{*}$ \\
\hline QRS, ms & $78.2 \pm 7.4$ & $80.2 \pm 9.9$ & $80.0 \pm 8.2$ & $82.3 \pm 9.4$ & $82.4 \pm 11.1$ & $82.3 \pm 10.7$ & $80.9 \pm 15.3$ \\
\hline QT, ms & $959.4 \pm 62.2$ & $924.6 \pm 100.7$ & $736.6 \pm 113.3^{*}$ & $624.2 \pm 89.8^{*}$ & $555.4 \pm 86.9^{*}$ & $488.7 \pm 33.2^{*}$ & $428.1 \pm 59.4^{*}$ \\
\hline $\mathrm{Mv}, \mathrm{mg}$ & $93.5 \pm 17.2$ & & & & & & \\
\hline $\mathrm{Cv}, \mathrm{mg}$ & $121.0 \pm 18.6$ & & & & & & \\
\hline
\end{tabular}

$* P<0.05$ - values vs sinus rhythm; $M v$ - ventricular mass; $C v$ - cardiac mass. 
Table 3.

Correlation (r) between the processes of depolarization and repolarization of VE in frogs

\begin{tabular}{|l|c|c|c|c|c|c|c|}
\hline \multirow{2}{*}{ Parameters } & \multirow{2}{*}{$\begin{array}{c}\text { Sinus } \\
\text { rhythm }\end{array}$} & \multicolumn{5}{|c|}{ HR (Hz) / Duration of cardiac cycle (ms) } \\
\cline { 3 - 8 } & HR, bpm & $0.6 / 1570$ & $0.7 / 1360$ & $0.8 / 1250$ & $0.9 / 1140$ & $1.0 / 1000$ & $1.1 / 937$ \\
\hline AT - RT & $38 \pm 7$ & 38 & 44 & 48 & 55 & 60 \\
\hline AT - ARI & $\mathrm{r}=0.072$ & $\mathrm{r}=0.043$ & $\mathrm{r}=0.021$ & $\mathrm{r}=-0.076$ & $\mathrm{r}=0.034$ & $\mathrm{r}=-0.033$ & $\begin{array}{c}\mathrm{r}=0.581 \\
P<0.05\end{array}$ \\
\hline RT - ARI & $\mathrm{r}=0.074$ & $\begin{array}{c}\mathrm{r}=-578 \\
P<0.05\end{array}$ & $\mathrm{r}=-0.031$ & $\begin{array}{c}\mathrm{r}=-0.567 \\
\mathrm{P}<0.05\end{array}$ & $\begin{array}{c}\mathrm{r}=-0.561 \\
P<0.05\end{array}$ & $\begin{array}{c}\mathrm{r}=-0.551 \\
P<0.05\end{array}$ & $\mathrm{r}=-0.012$ \\
\hline
\end{tabular}

The repolarization dispersion of epicardium on the whole, as well as repolarization of both epicardial sides separately, decreased under the higher rate $(P<0.05)$. The increasing HR from $0.6 \mathrm{~Hz}$ to $1.1 \mathrm{~Hz}$ led to the increased duration of ARIs on the ventral and dorsal fragments of VE as compared with initial sinus rhythm (Table 1). Additionally, the higher rate resulted in a different dorso-ventral distribution of the repolarization durations. We observed the shortest durations $(P<0.05)$ of ARIs on the dorsal fragment of VE compared to ventral side under the higher rate $(1.1 \mathrm{~Hz})$; repolarization duration decreased on VE of the frogs (Table 1).

Thus, the increased HR resulted in a decrease in the dispersion of repolarization and ARIs; the repolarization duration of VE at supraventricular rhythms was shortened as compared with sinus rhythm. During the high HR, more prolonged ARIs were observed on the ventral side of the epicardium than on the dorsal surface (exclusion is supraventricular rhythm with rate of $1.1 \mathrm{~Hz}$ ). The indexes of pump function decreased under high HR.

Under the sinus rhythm, we found a high positive correlation between the end of repolarization timing and the ARI duration, and the negative dependence of these parameters on AT (Table 3). Under HRs from $0.6 \mathrm{~Hz}$ to $1.1 \mathrm{~Hz}$, we found a positive correlation between the end of repolarization timing and durations of ARIs. Also, we observed a relationship between the time of depolarization and the end of repolarization timing at an HR of $1.1 \mathrm{~Hz}$. At the same time, we observed an inverse relationship between the AT and duration of repolarization at HRs of 0.6 and $0.8-1 \mathrm{~Hz}$.

Thus, under high HR, the repolarization sequence in VE of frogs does not repeat the activation sequence, especially, during sinus rhythm, and depends on the ARI distribution. An $\mathrm{HR}$ of $1.1 \mathrm{~Hz}$ is exclusion, where the repolarization sequence depends on the depolarization sequence and repolarization duration. During an increase in HR, the repolarization sequence is formed in association with the level of ARI dispersion and changes of the repolarization duration. The indexes of pump function decrease under high HR.

\section{Discussion}

HR in amphibians is directly related to the ambient temperature, and provides a fair estimate for the influence of ambient temperature on metabolism, ${ }^{[13,16]}$ as compared with endothermic vertebrates, when a high HR is a pivotal requirement for the high cardiac output needed to sustain the high metabolism associated with endothermy. ${ }^{[1,2]}$ In our research, the experiments were performed at $18-20^{\circ} \mathrm{C}$. It was shown, that all cardiomyocytes have already been activated at $18-20^{\circ} \mathrm{C}$, which corresponds to the preferred activity temperature of this species ectothermic vertebrates. ${ }^{[17]}$

In our study in frogs with increasing HR, we identified a slight increase in VEDP, and a significant decrease in systolic blood pressure and isovolumic indexes. According to R.Berger, the rapid atrial pacing of the heart led to potentially important mechanical alterations in canine heart. ${ }^{[6]}$ For example, as heart rate increases, atrial contraction occurs earlier in diastole and can become synchronous with the onset of LV filling. This could augment initial diastolic filling pressures and thereby influence pressures throughout a rate-limited diastolic period. Thus, the increased diastolic blood pressure in mammals as the HR increased led to the deterioration of coronary reserve and high energy phosphate metabolism, and, consequently, dysfunction of $\mathrm{Ca} 2+$ homeostasis in cardiomyocytes. ${ }^{[6]}$

It is known that regulation of the functional activity of the myocardium, rhythm and strength of heart contractions in frogs and mammals, is carried out with the participation of $\mathrm{Ca} 2+$, which generates a signal that triggers excitation and contraction of the heart.

Intracellular $\mathrm{Ca} 2+$ concentration is subjected to significant fluctuations, which has a modulating effect on the strength of heart contractions and HR. ${ }^{[18]}$ This effect is realized through a change in the action potential (AP) aimed at unloading cardiomyocytes at the excess load. It is possible that high HR in our study blocked $\mathrm{Ca} 2+$ - canals in cardiomyocytes during diastole, which has been accompanied by changes in the structural and functional organization of myocardium, directed at the support of vital activity in more extreme environmental conditions. As a result, the AP amplitude drops and myocardial activity decreases during systole, the value of which, in lower vertebrates, is determined by $\mathrm{Ca} 2+$ predominantly. [18] Therefore, we can assume that an increase in HR in frogs at poor development of $\mathrm{Ca} 2+$ channels of T-type and sarcoplasmic reticulum may be an indirect cause of reduction in cardiac isovolumic indexes and duration and dispersion of ARIs of VE, that we observed in our study. The $\mathrm{Na}+/ \mathrm{Ca} 2+-$ mechanism in frog cardiomyocytes operates to remove $\mathrm{Ca} 2+$ from cardiomyocytes. ${ }^{[18]}$ Under increased HR beyond the 
physiological norm for frogs, this mechanism seems to stop working, resulting in the phenomenon of dedifferentiation of cardiomyocytes and switches their activities to the fetal phenotype. The above-mentioned situation was observed in our study: the repolarization sequence depended on the activation sequence and the distribution of local repolarization durations only at supraventricular rhythm with a frequency of $1.1 \mathrm{~Hz}$.

The HR variability should be considered as a factor that provides the heart function in extreme conditions beyond the usual habitat of the organism. At the same time, we have shown that an increase in heart rate in amphibians is not accompanied by an increase in myocardial contractility. The natural variability of $\mathrm{HR}$ as a factor in increasing the contractile function is realized through an increase in the heterogeneity of the myocardium, whereas atrial artificial stimulation reduces the electrical heterogeneity of the myocardium. Most likely, the electric homogeneity of the myocardium is the reason for the change of contractile properties of the heart.

Thus, the increase in HR in amphibians is limited by a physiologically reasonable limit, specific to a particular type of animal, beyond which, the heart activity becomes ineffective and is accompanied by structural and functional remodeling. Also, initially, the structure and heterogeneity of the myocardium determine the maximal and functional properties of the heart in each cycle and the level of security of maximum HR.

\section{Acknowledgements}

The study was supported by the Ural Branch of the Russian Academy of Sciences (Project No. 12-P-4-1003).

\section{Competing interests}

The authors declare that they have no competing interests.

\section{References}

1. Jensen J, Wang T, Christoffels VM, Moorman AF. Evolution and development of the building plan of the vertebrate heart. Biochim Biophys Acta. 2013;1833(4):783-94.

2. Lillywhite HB, Zippel KC, Farrell AP. Resting and maximal heart rates in ectothermic vertebrates. Comp Biochem Physiol A Mol Integr Physiol. 1999;124(4):369-82.

3. Davies F, Francis ET. The conducting system of the vertebrate heart. Biol Rev Camb Phylos Soc. 1946;21(4):17388.

4. Moorman AF, Christoffels VM. Cardiac chamber formation: development, genes, and evolution. Physiol Rev. 2003; 83(4):1223-67.

5. Sedmera D, Reckova M, deAlmeida A, Sedmerova M, Biermann M, Volejnik J, et al. Functional and morphological evidence for a ventricular conduction system in zebrafish and Xenopus hearts. Am J Physiol Heart Circ Physiol. 2003;284(4):H1152-60.

6. Berger RD, Wolff MR, Anderson JH, Kass DA. Role of atrial contraction in diastolic pressure elevation induced by rapid pacing of hypertrophied canine ventricle. Circ Res. 1995; 77(1):163-73.

7. de Pauw M, Vilaine JP, Heyndrickx GR. Role of forcefrequency relation during AV-block, sinus node block and beta-adrenoceptor block in conscious animals. Basic Res Cardiol. 2004; 99 (5):360-71.

8. Vogel M, Cheung MM, Li J, Kristiansen SB, Schmidt MR, White PA, Sorensen K, et al. Noninvasive assessment of left ventricular force-frequency relationships using tissue Doppler-derived isovolumic acceleration: validation in an animal model. Circulation. 2003; 107(12):1647-52.

9. Weidermann F, Fadi J, Sutherland GR, Claus P, Kowalski M, Hatle L, et al. Myocardial function defined by strain rate and strain during alterations in inotropic states and heart rate. Am J Physiol Heart Circ Physiol. 2002;283(2):792-9.

10. Bowditch HP. Does the Apex of the Heart contract automatically? J Physiol. 1878; 1(1):104-7.

11. Carey C. Factors affecting body temperatures of toads. Oecologia. 1978; 35:197-219.

12. Branco LG, Wood SC. Effect of temperature on central chemical control of ventilation in the alligator Alligator mississippiensis. J Exp Biol. 1993;179:261-72.

13. Chapovetsky V, Katz U. Effects of season and temperature acclimation on electrocardiogram and heart rate of toads. Comp Biochem Physiol A Mol Integr Physiol. 2003; 134(1):77-83.

14. Mazza R, Pasqua T, Cerra MC, Angelone T, Gattuso A. Akt/eNOS signaling and PLN S-sulfhydration are involved in $\mathrm{H}_{2} \mathrm{~S}$-dependent cardiac effects in frog and rat. Am J Physiol Regul Integr Comp Physiol. 2013;305(4):443-51.

15. Millar CK, Kralios FA, Lux RL. Correlation between refractory periods and activation-recovery intervals from electrograms: effects of rate and adrenergic interventions. Circulation. 1985;72(6):1372-9.

16. Lagerspetz K. Interaction of season and temperatureacclimation in the control of metabolism in amphibian. Thermal Biol. 1977; 2:223-31.

17. Katz U, Hoffman J, Gil N. What is the ecological significance of laboratory temperature selection in anuran Amphybia? Alytes. 1997;15:91-8.

18. Shemarova IV, Kuznetsov SV, Demina IN, Nesterov VP. $\mathrm{T}$-channels and $\mathrm{Na}+, \mathrm{Ca} 2+$-exchangers as components of the $\mathrm{Ca} 2+$-system of the myocardial activity regulation of the frog Rana temporaria. Zh Evol Biokhim Fiziol. 2009;45(3):31928. [Article in Russian]. 